Journal for ImmunoTherapy of Cancer

\section{Leukemia escape in immune desert: intraocular relapse of pediatric pro-B- ALL during systemic control by CD19- CAR T cells}

To cite: Willier S, Raedler J, Blaeschke F, et al. Leukemia escape in immune desert: intraocular relapse of pediatric pro-B-ALL during systemic control by CD19-CAR T cells. Journal for ImmunoTherapy of Cancer 2020;8:e001052. doi:10.1136/jitc-2020-001052

SW and JR contributed equally.

Accepted 06 August 2020

Check for updates

(C) Author(s) (or their employer(s)) 2020. Re-use permitted under CC BY-NC. No commercial re-use. See rights and permissions. Published by BMJ.

For numbered affiliations see end of article.

\section{Correspondence to} Dr Tobias Feuchtinger; tobias.feuchtinger@med.unimuenchen.de

\section{ABSTRACT}

Background Relapsed/refractory B-precursor acute lymphoblastic leukemia (BCP-ALL) remains a major therapeutic challenge in pediatric hematology. Chimeric antigen receptor (CAR) T cells targeting CD19 have shown remarkable initial response rates in BCP-ALL patients, while long-term leukemia control rate is only about $50 \%$. So far, main mechanisms of BCP-ALL relapse after CD19CAR T-cell therapy have been either insufficient CAR T-cell persistence in vivo or loss of surface CD19.

Case Report Here, we report an exceptional presentation of BCP-ALL relapse in the eye during the systemic control through CAR T-cell therapy. We report a case of fatal intraocular relapse in a pediatric patient with pro-B-ALL after initial response to CD19-CAR T-cell therapy. One month after CD19-CAR T-cell therapy, remission was documented by bone marrow aspirate analysis with absence of CD19+ cells and CD19-CAR T cells could be detected in both peripheral blood and bone marrow. At the same time, however, the patient presented with progressive visual disturbance and $\mathrm{CD}_{1} 9^{+}$cells were found within the anterior chamber of the eye. Despite local and systemic therapy, ocular relapse led to BCP-ALL dissemination and systemic relapse within weeks. The eye represents a rare site for local manifestation of BCP-ALL, but isolated intraocular relapse is a clinically unreckoned presentation of BCP-ALL in the era of CD19-CAR T cells. Conclusion During systemic control of BCP-ALL through CD19-CAR T cells, relapse can emerge in the eye as an immune-privileged organ. Ocular symptoms after CD19CAR T-cell therapy should guide the clinician to elucidate the etiology in a timely fashion in order to adjust leukemia treatment strategy. Both, local immune escape as well as insufficient CAR T-cell persistence may have contributed to relapse in the reported patient. Mechanisms of relapse in an immune desert under CAR T-cell therapy require future clinical and experimental attention. In particular, ocular symptoms after CAR T-cell therapy should be considered a potentially early sign of leukemia relapse.

\section{BACKGROUND}

Relapsed/refractory B-precursor acute lymphoblastic leukemia (BCP-ALL) remains a major therapeutic challenge. Chimeric antigen receptor (CAR) T cells targeting CD19 have shown remarkable initial response rates but relevant relapse rates later on. ${ }^{12}$ Mechanisms of immune escape have been either loss of target structure or insufficient CAR T-cell persistence in vivo. ${ }^{34}$ Here, we report a novel clinical presentation of BCP-ALL relapse under CAR T-cell therapy, relapse within the eye as an immunologically privileged organ. CD19-CAR T cells are currently evaluated in numerous clinical trials ${ }^{5}$ and especially pediatric BCP-ALL shows initial response rates to CD19-CAR T cells of more than $80 \%$ in refractory or relapsed, heavily pretreated patients. ${ }^{1}$ Despite these promising response rates, about half of pediatric patients and the vast majority of adult patients experience relapse within 2years after CD19-CAR T-cell therapy. CD19 antigen loss under selective pressure is mediated through downregulation of CD19, splice variants/mutations of CD19 or myeloid differentiation of leukemic blasts. To our knowledge, an intraocular relapse has not been reported. The eye represents a sequestered compartment within the body that enjoys immune privilege through various immunomodulatory mechanisms such as immunosuppressant chemokine and ocular FasL expression. ${ }^{6}$ Extramedullary manifestations of pediatric ALL have been described in cerebrospinal fluid, testis and the eye and those manifestations are characterized as immune privileged sites. ${ }^{7}$ Here, we report the case of a 3-year-old boy with relapsed pro-B ALL and previous extensive extramedullary disease after two allogeneic hematopoietic stem cell transplantations (HSCT) and previous CD19-CAR T-cell therapy, who presented with an intraocular relapse after 
second CD19-CAR T-cell therapy, progressing to fatal systemic relapse.

\section{CASE PRESENTATION}

The boy was diagnosed with pro-B ALL, t(9;11) MLLrearrangement, central nervous system (CNS) involvement and initial white cell count of $30 \times 10^{\wedge} 9 / \mathrm{L}$ at 5 months of age. Minimal residual disease (MRD) markers persisted after initial treatment according to the Interfant-06 protocol $^{8}$ and a first HSCT from a HLA-compatible $(10 / 10)$ matched unrelated donor (conditioning with fludarabine, treosulfan, ATG-Grafalon) was performed at 12 months of age (figure 1A). Despite complete donor chimerism on day +55 , combined bone marrow $(\mathrm{BM})$ and CNS relapse occurred at week 12. Remission was achieved with blinatumomab in combination with pembrolizumab and the patient subsequently received a second HSCT with peripheral blood stem cells from his HLAhaploidentical mother at 16 months of age (conditioning with clofarabine, thiotepa, melphalan, ATG-Grafalon). A second combined relapse in BM and CNS 5 months after second HSCT proved refractory to blinatumomab and inotuzumab ozogamicin. At 27 months of age the patient received a first CD19-CAR T-cell therapy (4-1BB second generation CD19-CAR). BM analysis showed complete remission after 1 month. A CD $34^{+}$stem cell boost was performed for prolonged BM insufficiency.

Four months after CD19-CAR T-cell therapy, the patient suffered from a third combined BM and extramedullary relapse with a solitary skin lesion (immunohistochemistry $\mathrm{CD} 9^{+}$). A local irradiation with 3D-plan, $6 \mathrm{MeV}$ photons, to a total dose of $18 \mathrm{~Gy}(4 \times 4.5 \mathrm{~Gy})$ was carried out with good clinical response. Despite blinatumomab therapy, extramedullary leukemic progression occurred in testes and multiple subcutaneous chloromas. With CD19 still being expressed on leukemic cells of the patient after two lines of anti-CD19 therapy (blinatumomab and first CD19-CAR T-cell therapy), we expected the patient to benefit from a second CD19-CAR T-cell therapy. Consequently, treatment with tisagenlecleucel (Kymriah) was initiated at our institution with confirmed persistent $\mathrm{CD} 19^{+}$disease in the BM (figure 1B). After confirmation of complete donor chimerism in peripheral blood, lymphocyte apheresis of the patient was performed. No clinical symptoms of ocular pathology were present at that time point and cranial MRI with contrast medium did not show any ocular pathology, either. Second CD19-CAR T-cell treatment was performed after lymphocyte depletion with fludarabine and cyclophosphamide at 39 months of age. The patient received $8.7 \times 10^{6}$ cells per $\mathrm{kg}$ body weight, the CD4:CD8 ratio was 7.6:1 and the CD19-CAR transduction rate was $21 \%$ (figure $1 \mathrm{C}, \mathrm{D}$ ). The patient experienced cytokine release syndrome grade 1 on day +11 which was treated with nonsteroidal anti-inflammatory drugs. $\mathrm{CD}_{3}{ }^{+}$stem cell boost from the second HSCT donor was given for prolonged cellular aplasia complicated by bacterial sepsis. BM and CNS analysis on day +28 documented remission by microscopy, flow cytometry and genetic MRD testing (figure 1A,B). Moreover, CD19-CAR T cells could be detected in both peripheral blood and bone marrow by flow cytometry using FITC-labeled recombinant CD19 protein and by real-time PCR, although in low frequency $(\sim 1 \%$ of viable $\mathrm{T}$ cells).

On day +16 the patient displayed erythema of the left eye and was initially diagnosed with conjunctivitis. However, symptoms progressed with pain, hyposphagma, vision impairment, mild proptosis and ptosis within 1 week. Swab testing was negative for viral infection (adenovirus, cytomegalovirus (CMV) and Epstein-Barr virus) and local antibiotic therapy did not improve symptoms. On ophthalmological assessment on day +28 , the eye showed progressive conjunctival chemosis and anterior chamber cellular infiltration along with increased intraocular pressure (IOP). Intraocular bleeding was suspected due to thrombocytopenia. The patient was treated with platelet transfusion, systemic acetazolamide to reduce the IOP and local immunosuppressant dexamethasone. After progressive findings with hypopyon and worsening of the fundus reflex (figure 2A,B), biopsy of episclera and conjunctiva and an anterior chamber fluid tap with a single port vitrectomy for vitreous tap were performed.

Unfortunately, intraoperative findings and histology demonstrated episcleral and intraocular infiltration by atypical $\mathrm{CD} 19^{+}$lymphoid cells, confirming intraocular ALL relapse after second CD19-CAR T-cell therapy (figure 3). $\mathrm{CD}_{1}{ }^{+}$cells were found inside the lamina propria of the conjunctiva with a perivascular accumulation, whereas $\mathrm{CD}^{+}$cells were found preferentially within the epithelium of the conjunctiva (figure 2C). Immunohistochemistry of nucleated cells in the lavage material of the anterior chamber confirmed presence of $\mathrm{CD}_{1} 9^{+}$cells and complete absence of $\mathrm{CD}^{+}$cells inside the eye (figure 2D). The patient showed no signs of systemic or CNS relapse with absence of CD $19^{+}$ cells in BM (figure 1B), peripheral blood or cerebral spinal fluid. Prophylactic intrathecal chemotherapy was administered on day +37 (methotrexate, cytarabine, prednisolone) to prevent per continuitatem invasion of the CNS. Despite missing histological material from the right eye, clinical involvement with hyperemia led to the conclusion of a binocular disease. Subsequently, a bilateral orbital radiotherapy to a total dose of $20 \mathrm{~Gy}$ in ten fractions with $6 \mathrm{MeV}$ photon beam was initiated on day +40 as a palliative measure. Intraocular administration of chemotherapy was not performed. Radiotherapy was well tolerated with only minor local erythema and the patient showed temporary symptom relief. Unfortunately, the patient's condition deteriorated rapidly and peripheral blood testing confirmed systemic progression with leukocytosis $\left(34.5 \times 10^{\wedge} 9 / \mathrm{L}\right)$ and $51 \% \mathrm{CD} 19^{+}$peripheral blasts. Palliative chemotherapy with cytarabine was performed but the patient deceased 6 weeks after intraocular relapse on day +75 after second CD19-CAR T-cell therapy. 


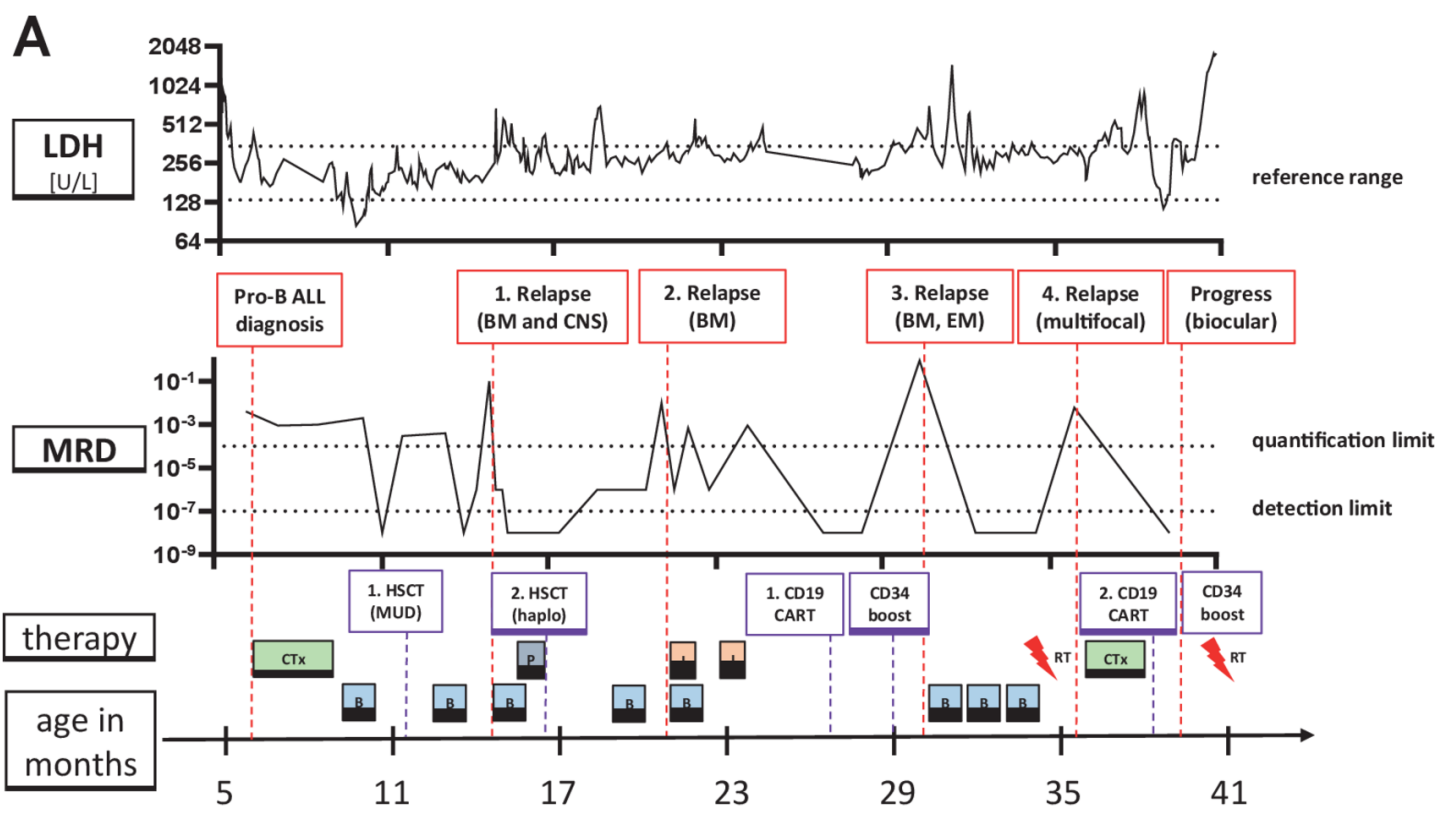

B 1st CD19 CART therapy 2nd CD19 CART therapy ocular relapse at D+28
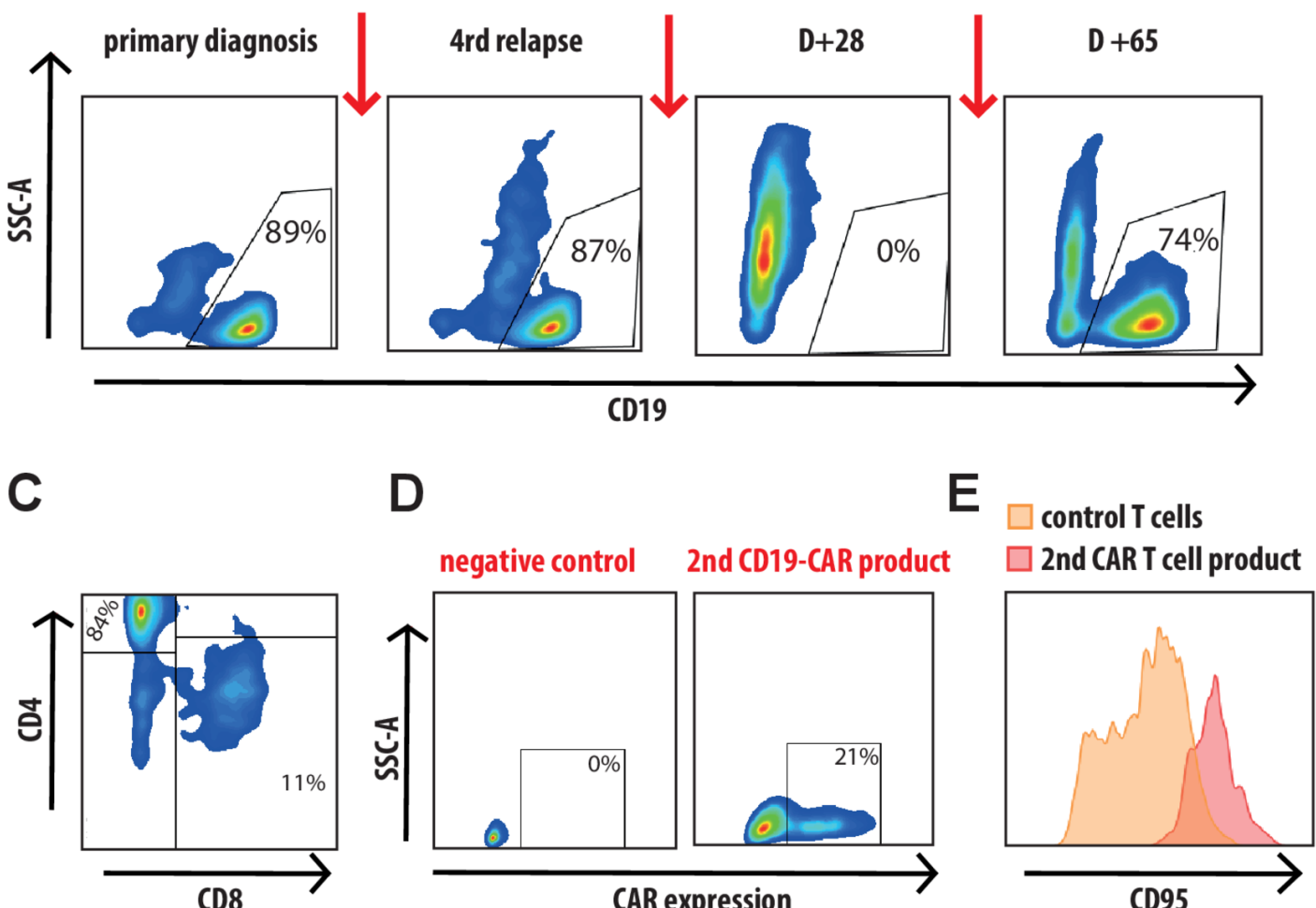

Figure 1 Clinical course. (A) LDH (lactate dehydrogenase) and MRD (genetic minimal residual disease) are displayed as leukemic markers. Red boxes indicate major events in the patient's history. (B) CD19 expression as measured by flow cytometry (clone SJ25C1, BD) was seen on bone marrow aspirates at initial diagnosis, third relapse and peripheral blood on day +65 after second CD19-CAR T-cell therapy. At the time of invasive ocular diagnostics, 12 days after ocular symptom onset on day +28 after second CD19-CAR T-cell therapy, no CD19 expression was detected within the bone marrow. (C) Flow cytometry analysis of second CD19-CAR product demonstrating a CD4:CD8 ratio of 7.6:1. (D) flow cytometry analysis of CD19-CAR transduction of second CD19-CAR product. FITC-labeled recombinant CD19 protein was used to detect CD19-CAR transduced CD3 ${ }^{+}$cells within the CAR product. A negative control using non-transduced T-cells is displayed as reference. (E) CD95 expression on $\mathrm{CD}^{+} \mathrm{T}$ cells in the second CD19-CAR product was measured by flow cytometry (red histogram, "second CAR T-cell product"). as reference, non-transduced T-cells were included in the histogram (orange histogram 'control T cells'). B, blinatumomab cycle; BM, bone marrow; CAR, chimeric antigen receptor; CNS, central nervous system; CTx, chemotherapy; HSCT, hematopoietic stem cell transplantation; I, inotuzumab ozogamicin cycle; P, pembrolizumab cycle; RT, radiotherapy; SSC-A: side scatter area; FITC: Fluorescein isothiocyanate. 


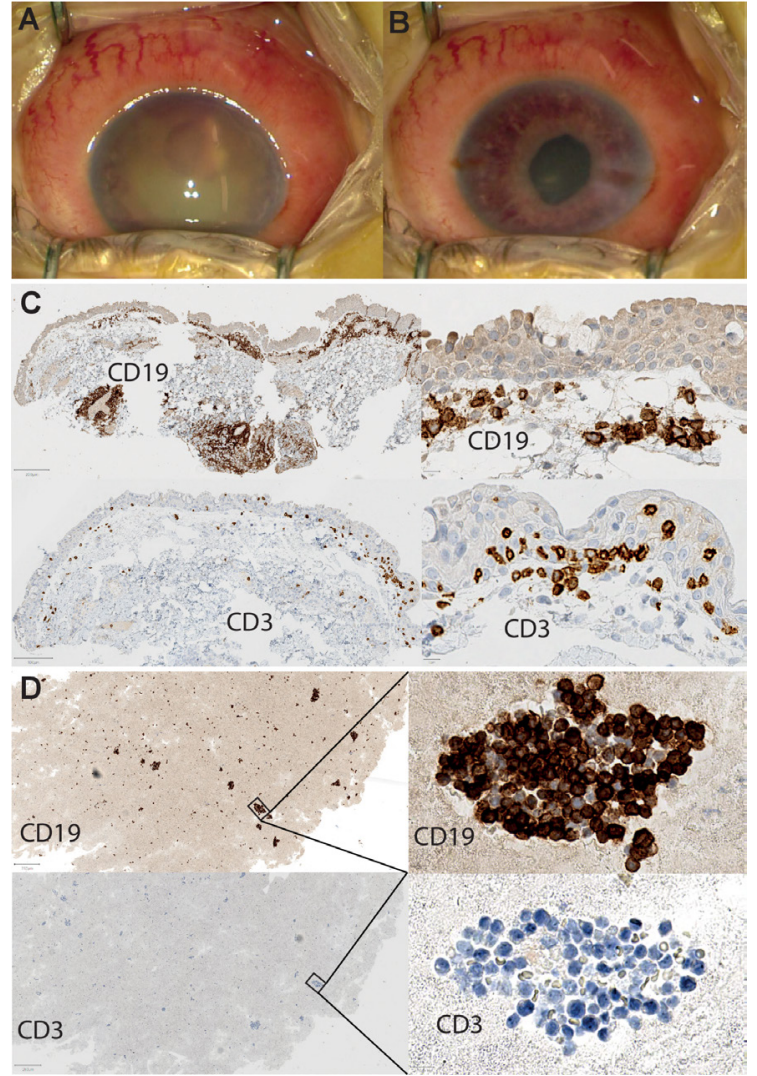

Figure 2 Clinical manifestation of binocular relapse of pro-B ALL after second CD19-CAR T-cell therapy. (A) Left eye before surgery. No intraocular structures are identifiable, the cornea appears clear but the anterior chamber is filled with a pus-like yellowish fluid, which resembles hypopyon in endophthalmitis. Note the hemorrhagic (reddish) appearance of the fluid which is not typically seen in infectious hypopyon. Additionally, the conjunctiva/sclera show a salmon-pink, 'fleshy' appearance, typically seen in conjunctival lymphoma. (B) Left eye after rinsing of the anterior chamber with extraction of the anterior chamber material. Now large dilated vessels on the surface of the iris can be identified at the periphery of the iris. The lens demonstrated a clear appearance. (C) CD3 and CD19 immunohistochemistry (IHC) of conjunctiva biopsy of the left eye. $\mathrm{CD} 3^{+} \mathrm{T}$ cells are concentrated at the surface of the conjunctiva. $\mathrm{CD} 19^{+}$cells show subepithelial localization and are widely observed within the connective tissue with additional perivascular accumulation. (D) CD3 and CD19 IHC from anterior ocular chamber lavage of the left eye. Most importantly, within anterior chamber lavage only $\mathrm{CD} 19^{+}$cells are present while in the CD3 IHC nucleated cells are CD3 negative. Black rectangles in left panel graphs illustrate the regions which are displayed in right panel graphs. for IHC, $4 \mu \mathrm{m}$ sections of formalin-fixed paraffin-embedded tissue blocks were cut and antigen retrieval was carried out with microwave treatment and antigen target retrieval solution (Agilent technologies) for $64 \mathrm{~min}(\mathrm{pH}$ 8.4). incubation for $28 \mathrm{~min}$ with either monoclonal rabbit anti-CD3 (1:150 dilution, clone Sp7, Zytomed) or monoclonal mouse anti-CD19 (1:20 dilution, clone EP169, Cellmarque) followed. Then, slides were incubated with a secondary anti-rabbit IgG antibody (MP-7401, ImmPress reagent kit, peroxidase-conjugated) followed by target detection using ultraView universal DAB detection kit (Ventana). BM, bone marrow; CAR, chimeric antigen receptor.
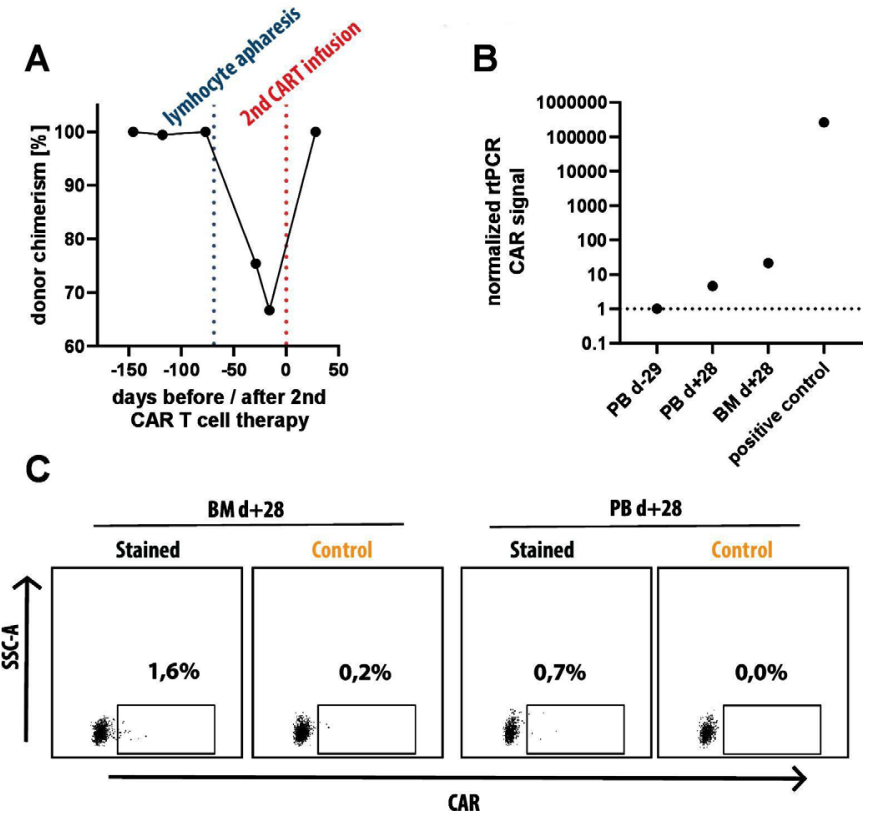

Figure 3 Donor chimerism at time of lymphocyte apheresis and detection of CD19 targeted CAR T cells at time of ocular relapse. (A) Donor chimerism in peripheral blood as determined by Fluorescence in situ analysis analysis based on $\mathrm{X} / \mathrm{Y}$ chromosome analysis with a female donor for a male patient. Shortly before lymphocyte apheresis from the patient (blue dotted line), 100\% donor chimerism was documented.

(B) Real-time PCR (RT-PCR) analysis of peripheral blood prior to second CAR T-cell therapy (PB d-29), peripheral blood (PB d+28) and bone marrow (BM d+28) 1 month after second CAR T-cell therapy and a positive control (in vitro generated CD19 CAR T-cells from our lab) using primers specific for the CAR molecule. A weak signal can be seen in both samples after second CAR T-cell therapy both in peripheral blood (4.6x negative control) and BM (21.5x negative control). (C) Flow cytometry analysis of the same two biosamples used for RT-PCR, taken at $d+28$ after second CAR T-cell therapy. after gating on lymphocytes (SSC-A vs FSC-A), single cells (FSC-H vs FSC-A), CD45 $5^{\text {hi }}, \mathrm{CD}^{+}$and TCRalpha/beta ${ }^{+}$we measured expression of CD19 CAR with recombinant, FITClabeled CD19 molecule (Stained) and used recombinant, FITC-labeled CD22 molecule as negative control (Control). within BM, $1.4 \%$ of CAR T cells can be detected and $0.7 \%$ CAR T cells are detected within peripheral blood. CAR, chimeric antigen receptor, FSC-A, forward scatter area, FSC, forward scatter height.

\section{DISCUSSION AND CONCLUSIONS}

In recent literature, CD19-CAR T cells have been shown to clear CNS disease after intravenous administration ${ }^{9}$ and a trial with 86 patients documented no effect of extramedullary disease prior to CD19-CAR T-cell therapy on prognosis. ${ }^{10}$ Ocular involvement of BCP-ALL at time of diagnosis is associated with CNS or BM relapse and a decrease in overall survival greater than $50 \% .{ }^{11}$ Glaucoma has been mentioned as possible symptom of ocular involvement. ${ }^{12}$ However, to our knowledge, there are no available data on frequency of extramedullary disease or ocular relapse after CD19-CAR T-cell therapy. 
The concept of immune privilege is an evolutionary adaptation to protect indispensable tissues and was first described in testes and eyes ${ }^{13-15}$ and more recently in other organs such as the $\mathrm{CNS}^{16}$ and is mediated by expression of immunosuppressant chemokines, FasL expression and absence of MHC-1 on ocular tissues. ${ }^{17}$ Moreover, it has been shown that CAR $\mathrm{T}$ cells are susceptible to FasL-induced cell death due to Fas (CD95) expression on CAR T cells. ${ }^{18}$ Indeed, CAR $\mathrm{T}$ cells administered prior to intraocular relapse showed high CD95 expression by flow cytometry (figure $1 \mathrm{E}$ ). We hypothesize that extramedullary-prone blasts and the immune privileged milieu within the eye may have led to immune evasion from CD19-CAR T-cell attack. This concept is supported by the simultaneous presence of $\mathrm{CD} 19^{+}$blasts within the anterior chamber of the left eye in complete absence of $\mathrm{T}$ cells while no $\mathrm{CD} 19^{+}$events could be detected within the $\mathrm{BM}$ or peripheral blood (figure 1, figure 2C,D). CD19-CAR $\mathrm{T}$ cells have been previously described to infiltrate immune privileged sites beyond the CNS, documented by, for example, a recent case report on bilateral retinal detachment after CD19-CAR T-cell therapy in a patient with retinal infiltration ${ }^{19}$ and by the efficacy of CD19-CAR T cells in testicular CD19+ ${ }^{+}$leukemia. ${ }^{20-22}$ In addition to local immune escape, mechanisms of insufficient CAR T-cell persistence may have led to relapse in our patient. The possible overlap and rapid evolvement of systemic leukemia after local relapse limit definitive mechanistic conclusions in our case. Future clinical and experimental studies will be required to clarify the mechanistic role of immune desert for relapse under selective pressure of CD19-CAR T cells.

Finally, we want to raise awareness for leukemia relapse after CD19-CAR T-cell therapy presenting with ocular symptoms resembling intraocular infection or thrombocytopenic hemorrhage. As most relevant differential diagnosis, intraocular infection may occur in immunecompromised patients, typically as CMV retinitis, or as bacterial or fungal infection. Intraocular relapse must be considered in patients presenting with ocular symptoms after CD19-CAR T-cell therapy, especially in patients with rapid symptom progression and elevated IOP. Accordingly, we suggest to initiate appropriate invasive diagnostic procedures in those patients, to clearly differentiate between leukemia-related and non-leukemiarelated etiology of ocular symptoms. Early detection and local treatment intervention might prevent fatal relapse progression.

\footnotetext{
Author affiliations

${ }^{1}$ Pediatric Hematology, Oncology, Hemostaseology and Stem Cell Transplantation, Dr. von Hauner Children's Hospital, University Hospital, LMU Munich, Munich, Bavaria, Germany

${ }^{2}$ German Cancer Research Center (DKFZ), Heidelberg, Germany

${ }^{3}$ Department of Radiotherapy, University Hospital Munich, Ludwig Maximilian University Munich, Munich, Germany

${ }^{4}$ Institute of Pathology, Faculty of Medicine, LMU Munich, Munich, Germany

${ }^{5}$ Max-Eder Research Group for Pediatric Sarcoma Biology, Institute of Pathology, Faculty of Medicine, LMU Munich, Munich, Germany
}

${ }^{6}$ Partner site Munich, German Cancer Consortium (DKTK), Munich, Germany ${ }^{7}$ Department of Ophthalmology, University Hospital Munich, Ludwig Maximilian University Munich, Munich, Germany

${ }^{8}$ Department of Ophthalmology, University of Ulm, Ulm, Germany

Acknowledgements The authors wish to thank the team at the von Hauner University Children's Hospital for the care of the patient, Gertrud und Hugo Adler Stiftung for support and Alexandra Skorupa (Novartis) for advice. SW was supported by the Else-Kröner-Fresenius Stiftung and DS by the German Cancer Consortium (DKTK).

Contributors The concept was set up by TF. The patient was treated by VB, MHA, IS, JR, SW, FB, AW, MPE and TF. TGPG performed immunohistochemistry and DS, FJ and SW performed flow cytometry. SW, JR and TF wrote the manuscript. All authors read and approved the final manuscript.

Funding The authors have not declared a specific grant for this research from any funding agency in the public, commercial or not-for-profit sectors.

\section{Competing interests None declared.}

Patient consent for publication Not required.

Ethics approval This study was performed in accordance with the Declaration of Helsinki and authorized by the local ethics committee (Ethikkommission der LMU München, project 17-163).

Provenance and peer review Not commissioned; externally peer reviewed.

Open access This is an open access article distributed in accordance with the Creative Commons Attribution Non Commercial (CC BY-NC 4.0) license, which permits others to distribute, remix, adapt, build upon this work non-commercially, and license their derivative works on different terms, provided the original work is properly cited, appropriate credit is given, any changes made indicated, and the use is non-commercial. See http://creativecommons.org/licenses/by-nc/4.0/.

\section{ORCID iDs}

Semjon Willier http://orcid.org/0000-0001-9472-2191

Tobias Feuchtinger http://orcid.org/0000-0002-8517-9681

\section{REFERENCES}

1 Maude SL, Laetsch TW, Buechner J, et al. Tisagenlecleucel in children and young adults with B-cell lymphoblastic leukemia. $N$ Engl $J$ Med 2018;378:439-48.

2 Chen L, Xu B, Long X, et al. Car T-cell therapy for a relapsed/ refractory acute $B$-cell lymphoblastic lymphoma patient in the context of Li-Fraumeni syndrome. J Immunother Cancer 2020;8:e000364.

3 Blaeschke F, Willier S, Stenger D, et al. Leukemia-induced dysfunctional $\mathrm{TIM}-3^{+} \mathrm{CD} 4^{+}$bone marrow $\mathrm{T}$ cells increase risk of relapse in pediatric B-precursor ALL patients. Leukemia 2020. doi:10.1038/s41375-020-0793-1. [Epub ahead of print: 13 Mar 2020].

4 Xu X, Sun Q, Liang X, et al. Mechanisms of relapse after CD19 CAR T-cell therapy for acute lymphoblastic leukemia and its prevention and treatment strategies. Front Immunol 2019;10:2664.

5 CLL - List Results. Search of: CD19 CAR T cells. Available: https:// clinicaltrials.gov/ct2/results?cond=CLL\&term $=C D 19+C A R+T+C e l l s \&$

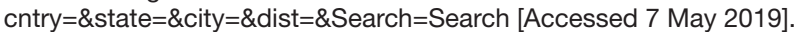

6 Griffith TS, Brunner T, Fletcher SM, et al. Fas ligand-induced apoptosis as a mechanism of immune privilege. Science 1995;270:1189-92.

7 Russo V, Scott IU, Querques G, et al. Orbital and ocular manifestations of acute childhood leukemia: clinical and statistical analysis of 180 patients. Eur J Ophthalmol 2008;18:619-23.

8 Pieters R, Schrappe M, De Lorenzo P, et al. A treatment protocol for infants younger than 1 year with acute lymphoblastic leukaemia (Interfant-99): an observational study and a multicentre randomised trial. The Lancet 2007;370:240-50.

9 Rheingold SR, Chen LN, Maude SL, et al. Efficient Trafficking of Chimeric Antigen Receptor (CAR)-Modified T Cells to CSF and Induction of Durable CNS Remissions in Children with CNS/ Combined Relapsed/Refractory ALL. Blood 2015;126:3769.

10 Zhang X, Lu X-an, Yang J, et al. Efficacy and Safety of CD19 Chimeric Antigen Receptor (CAR) T Cell Therapy for B-Cell Acute Lymphocytic Leukemia (B-cell ALL) in a Large Cohort Including Patients with Extramedullary Disease(EMD), High Leukemia Burden, BCR-ABL (+) Mutation,TP53 Mutation, and Post-Transplant Relapse. Blood 2018;132:280. 
11 Ohkoshi K, Tsiaras WG. Prognostic importance of ophthalmic manifestations in childhood leukaemia. $\mathrm{Br} J$ Ophthalmol 1992;76:651-5.

12 Sharma T, Grewal J, Gupta S, et al. Ophthalmic manifestations of acute leukaemias: the ophthalmologist's role. Eye 2004;18:663-72.

13 Niederkorn JY, Streilein JW. Lymphoma allografts abrogate immune privilege within the anterior chamber of the eye. Invest Ophthalmol Vis Sci 1986;27:1235-43.

14 Head JR, Neaves WB, Billingham RE. Immune privilege in the testis. Transplantation 1983;36:423-30.

15 Benhar I, London A, Schwartz M. The privileged immunity of immune privileged organs: the case of the eye. Front Immunol 2012;3:296.

16 Niederkorn JY. See no evil, hear no evil, do no evil: the lessons of immune privilege. Nat Immunol 2006;7:354-9.

17 Abi-Hanna D, Wakefield D, Watkins S. Hla antigens in ocular tissues. I. in vivo expression in human eyes. Transplantation 1988;45:610-3.
18 Tschumi BO, Dumauthioz N, Marti B, et al. Cart cells are prone to Fas- and DR5-mediated cell death. J Immunother Cancer 2018;6:71.

19 Denton CC, Gange WS, Abdel-Azim H, et al. Bilateral retinal detachment after chimeric antigen receptor T-cell therapy. Blood Adv 2020;4:2158-62.

20 Chen X, Wang Y, Ruan M, et al. Treatment of testicular relapse of B-cell acute lymphoblastic leukemia with CD19-specific chimeric antigen receptor T cells. Clin Lymphoma Myeloma Leuk 2020;20:366-70.

$21 \mathrm{Yu}$ J, Hu Y, Pu C, et al. Successful chimeric Ag receptor modified T cell therapy for isolated testicular relapse after hematopoietic cell transplantation in an acute lymphoblastic leukemia patient. Bone Marrow Transplant 2017;52:1065-7.

22 Ping N, Qu C, Bai L, et al. Successful chimeric antigen receptor $T$ cell therapy in a case of primary testicular diffuse large-B-cell lymphoma with central nervous system progression. Leuk Lymphoma 2019;60:2814-6. 